RESEARCH ARTICLE

\title{
Use of KDP crystal as a Kerr nonlinear medium for compressing PW laser pulses down to 10 fs
}

\author{
Andrey Shaykin, Vladislav Ginzburg, Ivan Yakovlev, Anton Kochetkov, Alexey Kuzmin, Sergey Mironov, \\ Ilya Shaikin, Sergey Stukachev, Vladimir Lozhkarev, Artem Prokhorov, and Efim Khazanov \\ Institute of Applied Physics of the Russian Academy of Sciences, Nizhny Novgorod 603950, Russia \\ (Received 9 August 2021; revised 27 August 2021; accepted 3 September 2021)
}

\begin{abstract}
The input pulse of the laser PEARL with energy of $18 \mathrm{~J}$ and pulse duration of about $60 \mathrm{fs}$ was compressed to $10 \mathrm{fs}$ after passage through a 4-mm-thick KDP crystal and reflection at two chirped mirrors with sum dispersion of $-200 \mathrm{fs}^{2}$. The experiments were performed for the $B$-integral values from 5 to 19 without visible damage to the optical elements, which indicates that small-scale self-focusing is not a significant issue. It was shown that, by virtue of the low dispersion of the group velocity, the KDP crystal has some advantages over silica: a larger pulse compression coefficient, especially at a small value of the $B$-integral $(B=5, \ldots, 9)$, lower absolute values of chirped mirror dispersion, and also a possibility to control the magnitude of nonlinearity and dispersion by changing crystal orientation.
\end{abstract}

Keywords: CafCA; petawatt lasers; post-compression; self-phase modulation; small-scale self-focusing; thin film compression; third-order nonlinearity

\section{Introduction}

The power and, hence, the focal intensity of petawatt and multipetawatt lasers are currently limited by the size and damage threshold of compressor diffraction gratings ${ }^{[1]}$. A multifold power enhancement can be achieved using mosaic gratings in the compressor or several parallel chirped pulse amplification (CPA) channels, each of which ends with a conventional compressor of its own. In these cases, the power of the pulse increases as a result of increasing energy, while its duration remains unchanged. This approach entails a multiple increase in complexity, size, and price. An alternative recently developing approach, within the framework of which power is enhanced due to reduced pulse duration after the compressor, rather than due to energy increase, is free from these drawbacks. This approach has different names/acronyms: thin film compression (TFC) ${ }^{[2]}$, compression after compressor approach $(\mathrm{CafCA})^{[3]}$, or postcompression $^{[4]}$. The corresponding technique is as follows: the pulse spectrum is broadened as a result of self-phase modulation (SPM) during propagation in a medium with

Correspondence to: E. Khazanov, Institute of Applied Physics of the Russian Academy of Sciences, Nizhny Novgorod 603950, Russia. Email: efimkhazanov@gmail.com
Kerr nonlinearity and is then compressed due to reflection from chirped mirrors (CMs) with negative dispersion.

The idea to use cubic nonlinearity for SPM was proposed as early as in $1969^{[5]}$. The compression of a 20 ps pulse by several times was demonstrated in Ref. [6]. Later, nonlinear compression was obtained in the femtosecond range in a fiber $^{[7]}$, in hollow waveguides filled with gas ${ }^{[8]}$, and in bulk material bounded in the transverse direction ${ }^{[9]}$. Despite a huge number of works (see, for example, references in the review paper Ref. [3]), pulses were compressed only with millijoule energy, and the energy efficiency was less than $50 \%$. In the recent years, quite a few experimental results were obtained ${ }^{[10-17]}$, in which CafCA was successfully implemented for pulses with an energy of 1-18 J and energy efficiency close to $100 \%$. An important impetus for these studies was the proposed and experimentally confirmed method of suppressing small-scale self-focusing ${ }^{[18,19]}$, even at large values of the $B$-integral

$$
B=\left(2 \pi / \lambda_{0}\right) L n_{2} I_{\text {in }},
$$

where $n_{2}$ and $L$ are, respectively, the nonlinear index of refraction and the thickness of the nonlinear medium, $\lambda_{0}=2 \pi c / \omega_{0}$ is the central wavelength in vacuum, and $I_{\text {in }}$ is the input peak intensity. In particular, in Ref. [17] the 
experiments were performed for the $B$-integral values up to 19. Large values of $B$-integral enable a significant increase of the compression ratio. Also noteworthy are the demonstration of two-stage compression ${ }^{[20,21]}$ and the theoretical works on detailed numerical modeling ${ }^{[2,22,23]}$ and on expanding the capabilities of the technique aimed at simultaneous contrast enhancement ${ }^{[24-28]}$.

In experiments, different materials were used as a nonlinear medium, e.g., glass ${ }^{[10]}$, polyethylene terephthalate ${ }^{[11]}$, but in the majority of works it was silica ${ }^{[12-17]}$. As shown in Ref. [3], a compressed pulse will be shorter at a minimal ratio of the group velocity dispersion $k_{2}$ to the nonlinear index of refraction $n_{2}$. A decrease in $k_{2} / n_{2}$ is especially important for large $B$-integral values at which pulse spectrum is broadened markedly by SPM. Among the materials that can be used for SPM in high-power lasers, silica and BK7 glass have a small value of $k_{2} / n_{2}$ : approximately $1 \mathrm{fs}^{2} \mathrm{PW} / \mathrm{mm}^{3}$ at a wavelength of $910 \mathrm{~nm}$. For many glasses, isotropic and anisotropic crystals, $k_{2} / n_{2}$ is in the $1.5-2.5 \mathrm{fs}^{2} \cdot \mathrm{PW} / \mathrm{mm}^{3}$ range. An exception is a KDP crystal (ordinary wave), as its dispersion at a wavelength of $910 \mathrm{~nm}$ is only $11.3 \mathrm{fs}^{2} / \mathrm{mm}$, which is a factor of 2.5 less than that of silica while $n_{2}$, in contrast, is higher than in silica; for KDP $k_{2} / n_{2}=0.25 \mathrm{fs}^{2} \cdot \mathrm{PW} / \mathrm{mm}^{3}$.

In the presented work we experimentally studied the compression of an output pulse of the laser PEARL (PEtawatt pARametric Laser ${ }^{[29]}$ ) by SMP in a KDP crystal in a wide range of $B$-integral values from 5 to 19 and compared the obtained results with those using silica ${ }^{[15,17,20]}$.

\section{Features of nonlinear compression with the use of KDP}

All the aspects of nonlinear compression (spectrum broadening, pulse shortening, and peak intensity increase) were considered in detail in Ref. [3] as a function of two key parameters: the $B$-integral (Equation (1)) and the dimensionless parameter of nonlinear medium dispersion

$$
D=L \frac{k_{2}}{T_{\mathrm{F}}^{2}}
$$

where $T_{\mathrm{F}}$ is the full width at half maximum (FWHM) duration of the input Fourier-transform-limited (FTL) Gaussian pulse. The parameters $B$ and $D$ characterize nonlinearity and dispersion and have a simple physical meaning: $B$ is the ratio of the medium length $L$ to the nonlinear length (the length at which a nonlinear phase equal to 1 is accumulated) and $D$ is the ratio of $L$ to the dispersion length $L_{\mathrm{d}}=T_{\mathrm{F}}^{2} / k_{2}$. The KDP crystal at the wavelength $\lambda=910 \mathrm{~nm}$ has a very small value of $k_{2}=11.3 \mathrm{fs}^{2} / \mathrm{mm}$. Hereinafter, we use the Sellmeier equation from Ref. [30]. Moreover, $k_{2}$ reduces with growing $\lambda$ and at $\lambda=985 \mathrm{~nm}$ changes its sign. Consequently, not only group velocity dispersion $k_{2}$ but also the next, third-order dispersion (TOD) should be taken into account. For silica, the value of $k_{2}$ is much higher $\left(28 \mathrm{fs}^{2} / \mathrm{mm}\right)$. However, as we show in the following, at large $B$ values the effect of TOD is even more significant.

With allowance for TOD, the laser pulse propagation in a medium with Kerr nonlinearity is described by the equation

$\frac{\partial a}{\partial \xi}-\frac{i \cdot D}{2} \frac{\partial^{2} a}{\partial \eta^{2}}+\frac{T}{6} \frac{\partial^{3} a}{\partial \eta^{3}}=-i B\left(|a|^{2} \cdot a-\frac{2 \cdot i}{\pi \cdot N} \frac{\partial}{\partial \eta}\left(|a|^{2} \cdot a\right)\right)$,

where $\xi=z / L, z$ is the longitudinal coordinate, $L$ is the thickness of the plate, $\eta=(t-z / u) / T_{\mathrm{F}}, \frac{1}{u}=\left.\frac{\partial k(\omega)}{\partial \omega}\right|_{\omega=\omega_{0}}, a=A / A_{10}$ is the complex amplitude of the field normalized to the value at the input boundary $\left(A_{10}\right), T=L k_{3} / T_{\mathrm{F}}{ }^{3}$ is the dimensionless parameter TOD, $k_{3}=\left.\frac{1}{2 k_{0}} \frac{\partial^{3} k(\omega)^{2}}{\partial \omega^{3}}\right|_{\omega=\omega_{0}}, k(\omega)=n(\omega) \omega / c$ is the wave vector, $k_{0}=k\left(\omega_{0}\right), N=T_{\mathrm{F}} / \tau$, and $\tau=2 \pi / \omega_{0}$ is the period of the optical field. At the input of the nonlinear medium, let there be a Gaussian FTL pulse

$$
a(t, z=0)=e^{-2 \cdot \ln (2) \cdot \frac{t^{2}}{T_{\mathrm{F}}^{2}}}
$$

CMs introduce a quadratic spectral phase; thereby the field amplitude of the output (compressed) pulse $a_{\text {out }}$ is defined by

$$
a_{\mathrm{out}}(t)=F^{-1}\left(e^{-\frac{i \alpha \cdot \Omega^{2}}{2}} \cdot F(a(t, z=L))\right)
$$

where $\Omega=\omega-\omega_{0}, \alpha$ is the parameter of CM dispersion, and $F$ and $F^{-1}$ are the direct and inverse Fourier transforms. The quantity $\alpha_{\text {opt }}$ designates the value of $\alpha$ at which the compressed pulse intensity $I_{\text {out }}$ is maximal, and hence, the intensity increase factor $F_{\mathrm{i}}=I_{\mathrm{out}} / I_{\mathrm{in}}$ is also maximal. The numerical modeling (Equations (3)-(5)) was performed for KDP and silica with the parameters given in Table 1. Values of $B$ were varied from 0 to 20 by changing the input pulse intensity $I_{\text {in }}$. Here $B=10$ corresponds to an intensity of $0.79 \mathrm{TW} / \mathrm{cm}^{2}$ for KDP and $1.18 \mathrm{TW} / \mathrm{cm}^{2}$ for silica. Curves for $\alpha_{\text {opt }}(B)$ are plotted in Figure 1(a). The dashed curves for $k_{3}=0$ are given for comparison. Analogous curves for $F_{\tau}=\tau_{\text {in }} / \tau_{\mathrm{o}}$ and $F_{\mathrm{i}}$ at $\alpha=\alpha_{\mathrm{opt}}$ are plotted in Figures 1(b)

\begin{tabular}{|c|c|c|c|}
\hline Parameter & Units & KDP & Silica \\
\hline L & $\mathrm{mm}$ & 4 & 5 \\
\hline$k_{2}$ & $\mathrm{fs}^{2} / \mathrm{mm}$ & 11.3 & 27.7 \\
\hline$D$ & & 0.01 & 0.037 \\
\hline$k_{3}$ & $\mathrm{fs}^{3} / \mathrm{mm}$ & 123.7 & 106 \\
\hline$T$ & & 0.0022 & 0.0023 \\
\hline$n_{2}$ & $10^{-16} \mathrm{~cm} / \mathrm{W}$ & 4.6 & 2.45 \\
\hline$\lambda_{0}$ & $\mathrm{~nm}$ & \multicolumn{2}{|c|}{910} \\
\hline$T_{\mathrm{F}}$ & fs & \multicolumn{2}{|c|}{61} \\
\hline$N$ & & \multicolumn{2}{|c|}{20.1} \\
\hline
\end{tabular}
and 1(c).

Table 1. The parameters of numerical modeling. 

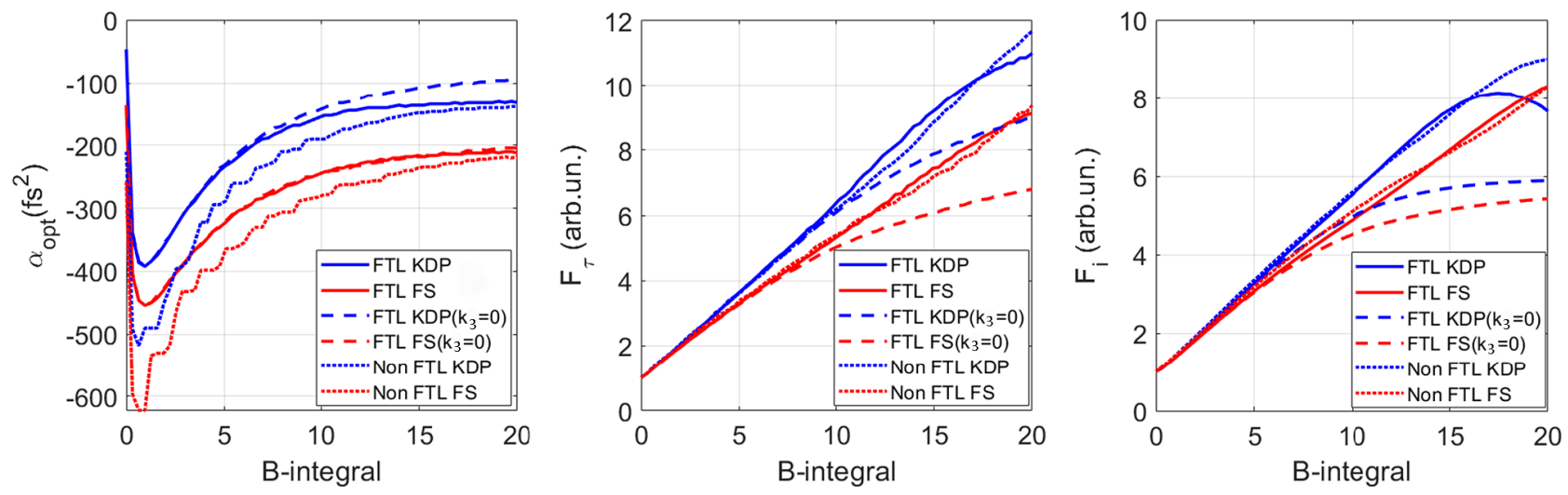

Figure 1. Curves for (a) $\alpha_{\mathrm{opt}}(B)$, (b) $F_{\tau}(B)$, and (c) $F_{\mathrm{i}}(B)$ for $\alpha=\alpha_{\mathrm{opt}}$ for KDP (blue) and silica (red): FTL pulse at $k_{3} \neq 0$ (solid curves) and $k_{3}=0$ (dashed curves); non-Gaussian FTL pulse, the spectrum and autocorrelation function of which are presented in Figure 3(a) (dotted curves). FTL, Fourier-transformlimited.

It is clear from Figure 1(a) that at large $B$ the allowance for TOD in KDP (in contrast to silica) results in an increase in the absolute value of $\alpha_{\text {opt }}$, which should be taken into consideration when planning experiments. In addition, with TOD taken into account, both $F_{\tau}$ and $F_{\mathrm{i}}$ become much larger for both KDP and silica. This is quite unexpected. Physically this is explained by the fact that at SPM the pulse acquires not only a positive quadratic spectral phase that is compensated by the negative quadratic dispersion of CMs, but also a negative cubic spectral phase that is partially compensated for by the positive cubic dispersion of the medium. As the values of $T$ are identical for KDP and silica (see Table 1), the effect of TOD on these media is approximately the same (see Figures 1(a)-1(c)).

\section{Experimental results}

The schematic diagram of the experiment is shown in Figure 2. After reflection from the last diffraction grating of the compressor, the PEARL laser beam (central wavelength of about $910 \mathrm{~nm}$ ) with a pulse energy of up to $18 \mathrm{~J}$, a duration of 55-67 fs, and a diameter of $18 \mathrm{~cm}$ propagated $2.5 \mathrm{~m}$ in free space for self-filtering ${ }^{[18]}$. After that, the beam was propagated in a 4-mm-thick homemade KDP crystal with angles of optical axes $\theta=\varphi=0$. The output surface had an anti-reflecting coating. We used the reflection from the input uncoated surface to measure the spectrum and the autocorrelation function (ACF) of the input pulse. The measurements were made for a small part of the beam with a diameter of $1 \mathrm{~cm}$. After free propagation over a distance of $6 \mathrm{~m}$, the beam was reflected from CMs with a diameter of $20 \mathrm{~cm}$ manufactured by UltraFast Innovations $\mathrm{GmbH}$ (reflection coefficient $>99 \%$, bandwidth $>200 \mathrm{~nm}$ ). We used mirrors with sum dispersion $\alpha=-100 \mathrm{fs}^{2},-200 \mathrm{fs}^{2}$, and $-250 \mathrm{fs}^{2}$. In addition, in the experiments with $\alpha=-200 \mathrm{fs}^{2}$ and $-250 \mathrm{fs}^{2}$ we placed a $1-\mathrm{mm}$-thick silica plate before the autocorrelator to introduce additional dispersion $\alpha=+28 \mathrm{fs}^{2}$, which is equivalent to the sum CMs dispersion $\alpha=-172 \mathrm{fs}^{2}$ and $-222 \mathrm{fs}^{2}$, respectively.

To measure the parameters of the output (compressed) pulse, a glass wedge (GW) with an aperture of $1 \mathrm{~cm} \times$ $2 \mathrm{~cm}$ and a matt back surface was placed in the beam path. The beam reflected from the first surface of the wedge was directed to the spectrometer and the autocorrelator. The position of the wedge within the beam aperture corresponded to the place where the ACF and the spectrum of the input beam were measured, which made it possible to measure the characteristics of the input and output pulses in a single shot. We used 1.42 as a decorrelation factor to calculate the pulse duration from the autocorrelation function.

Typical measurement results are presented in Figure 3 for two shots at $\alpha=-200 \mathrm{fs}^{2}$. The output pulse spectra have characteristic narrow peaks arising because the input pulses are not FTL (see Ref. [23] for details). Note that the measured output pulse spectrum is bounded at the longwave side by the spectrometer bandwidth (1030 nm).

An yoptimal value of CMs dispersion $\alpha_{\text {opt }}$ depends on $B$ (see Figure 1(a)). The curves for the minimal duration of a compressed pulse $\tau_{\text {out }}$ as a function of $\alpha$ for two ranges $B=5, \ldots, 9$ and $B=11, \ldots, 19$ are plotted in Figure 4. Analogous curves for 5-mm-thick and 3-mm-thick silica $^{[15,20]}$ are also presented in this figure. Note that these are qualitative data, as a different number of shots were made for different values of $\alpha$. Nevertheless, some conclusions follow from Figure 4. First, the value of $\alpha_{\text {opt }}$ decreases with a decrease in the parameter $D: \alpha_{\text {opt }}$ is maximal for 5-mmthick silica ( $D=0.037$ at $\left.\tau_{\text {in }}=61 \mathrm{fs}\right)$ and minimal for KDP $\left(D=0.01\right.$ at $\tau_{\text {in }}=61 \mathrm{fs}$ ). The difference is not very large for large values of $B$, but is significant for small $B$. This fully agrees with Figure 1(a). Second, the absolute values of $\alpha_{\text {opt }}$ obtained experimentally are larger than those predicted theoretically (see Figure 1(a)). The reason is that the pulse used in experiments was not an FTL pulse. The absolute 


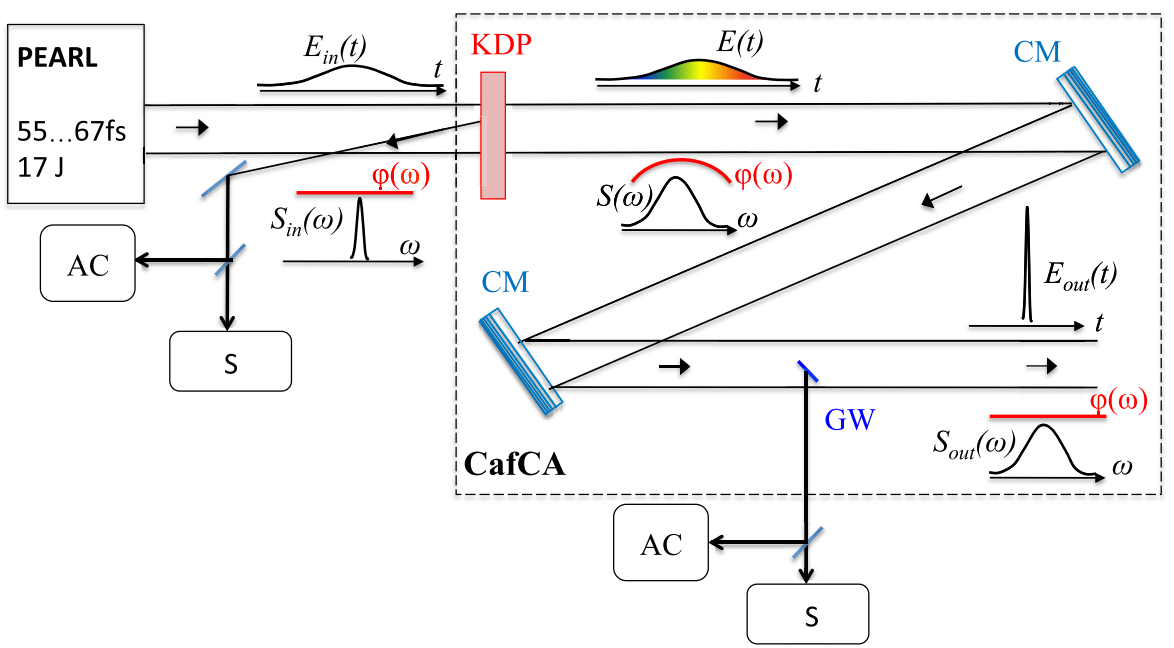

Figure 2. Schematic of the experiment. CM, chirped mirror; GW, small-aperture glass wedge; AC, autocorrelator; S, spectrometer.

a
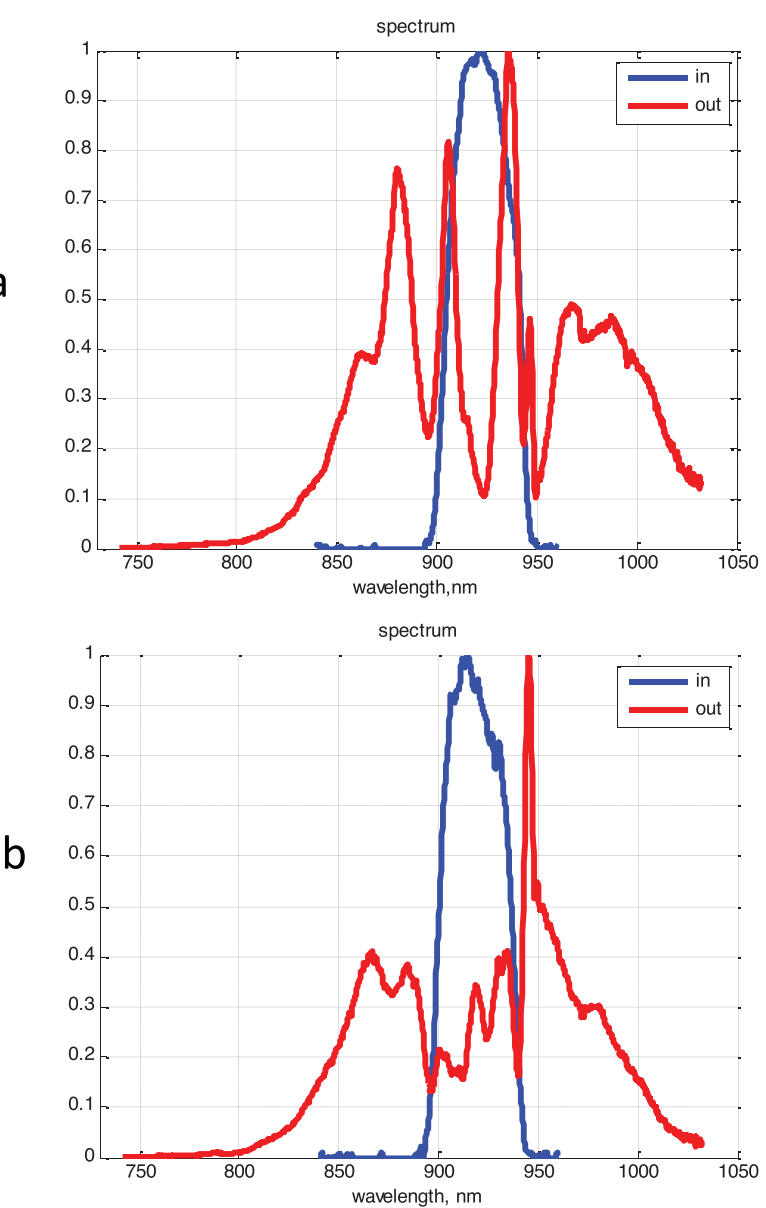

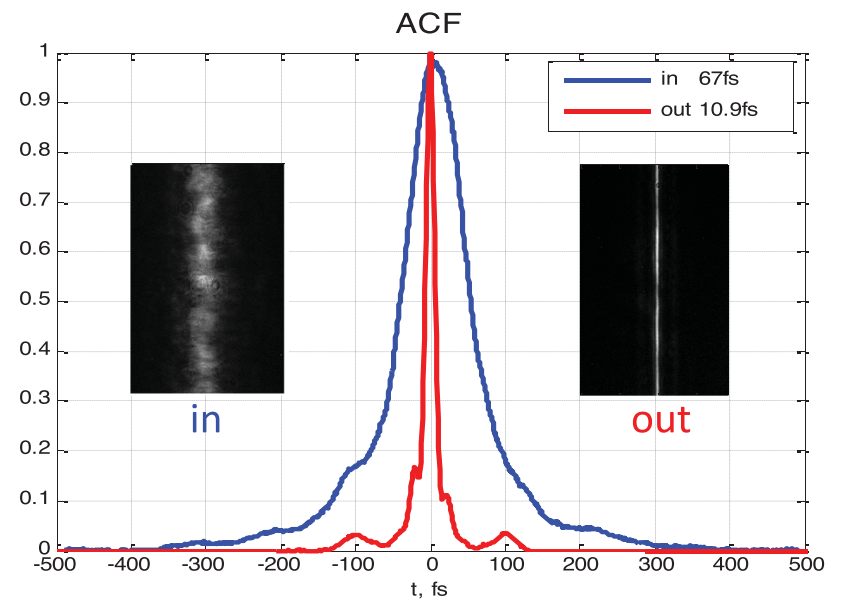

ACF

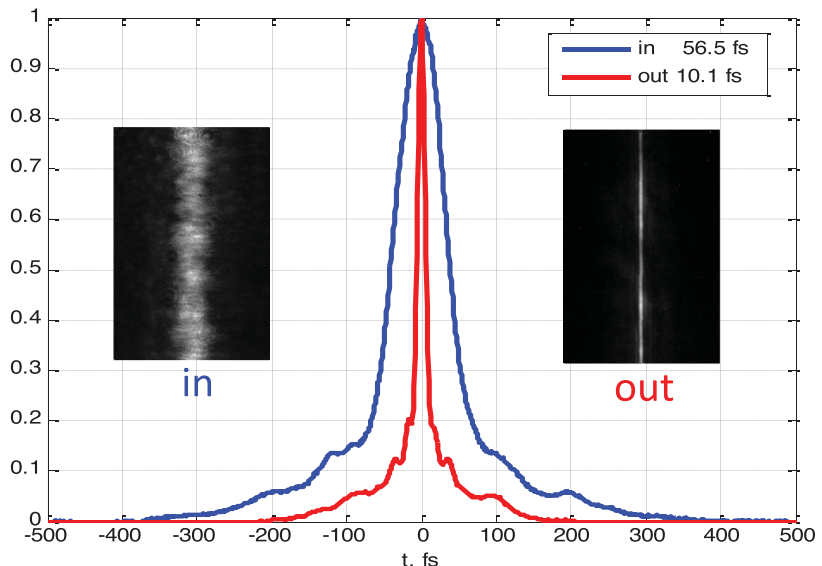

Figure 3. Measured input (blue) and output (red) spectra and ACF for two typical shots: (a) $B=13, \tau_{\text {in }}=67 \mathrm{fs}, \tau_{\text {out }}=10.9 \mathrm{fs}$ and (b) $B=14, \tau_{\text {in }}=57 \mathrm{fs}$, $\tau_{\text {out }}=10.1 \mathrm{fs}$.

value of $\alpha_{\text {opt }}$ for such pulses is much larger than for FTL pulses, which was first noted in Ref. [11]. To illustrate this fact, we presented in Figure 1(a) an $\alpha_{\text {opt }}(B)$ curve for a KDP crystal for the case when the input pulse has the spectrum and ACF shown in Figure 3(a): the dotted blue curve is lower than the solid curve. Third, from the viewpoint of minimal pulse duration, KDP, although slightly, is preferable to silica: 10 versus $11 \mathrm{fs}$ at large values of $B$ and 13 versus $16 \mathrm{fs}$ at small $B$. This also agrees with the results of numerical modeling (see Figure 1). 


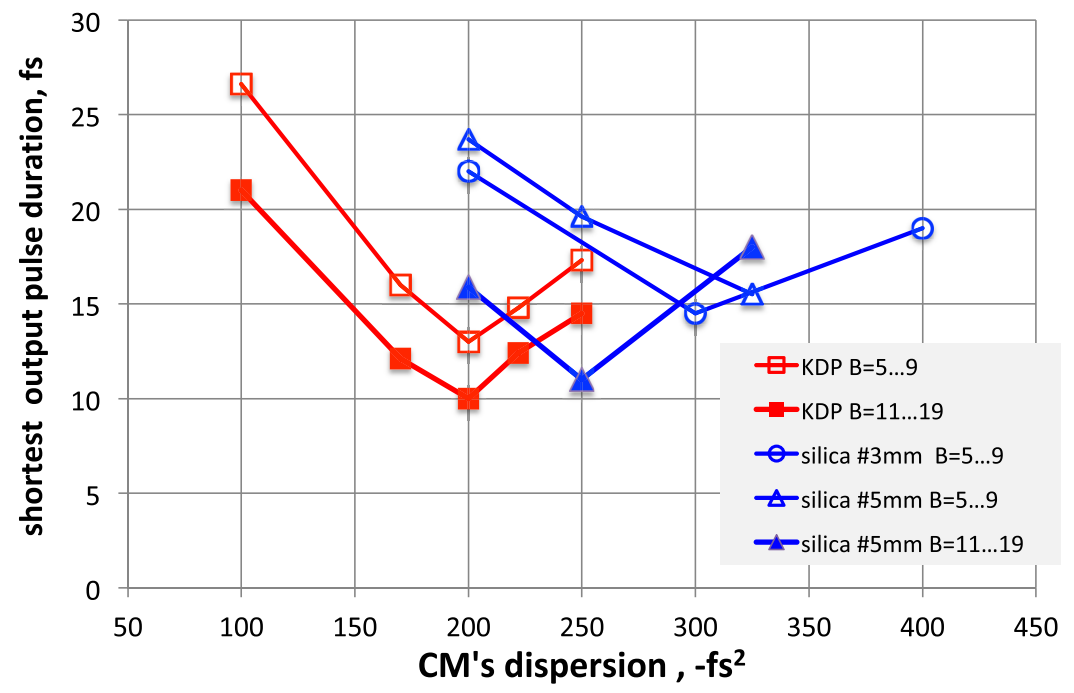

Figure 4. Experimental minimal compressed pulse duration $\tau_{\text {out }}$ for $\mathrm{KDP}(L=4 \mathrm{~mm})$, silica $(L=5 \mathrm{~mm})$, and silica $\left(L=3 \mathrm{~mm}^{[15,20]}\right)$ for two ranges of $B$ values. The curves are plotted to make the figure more illustrative.

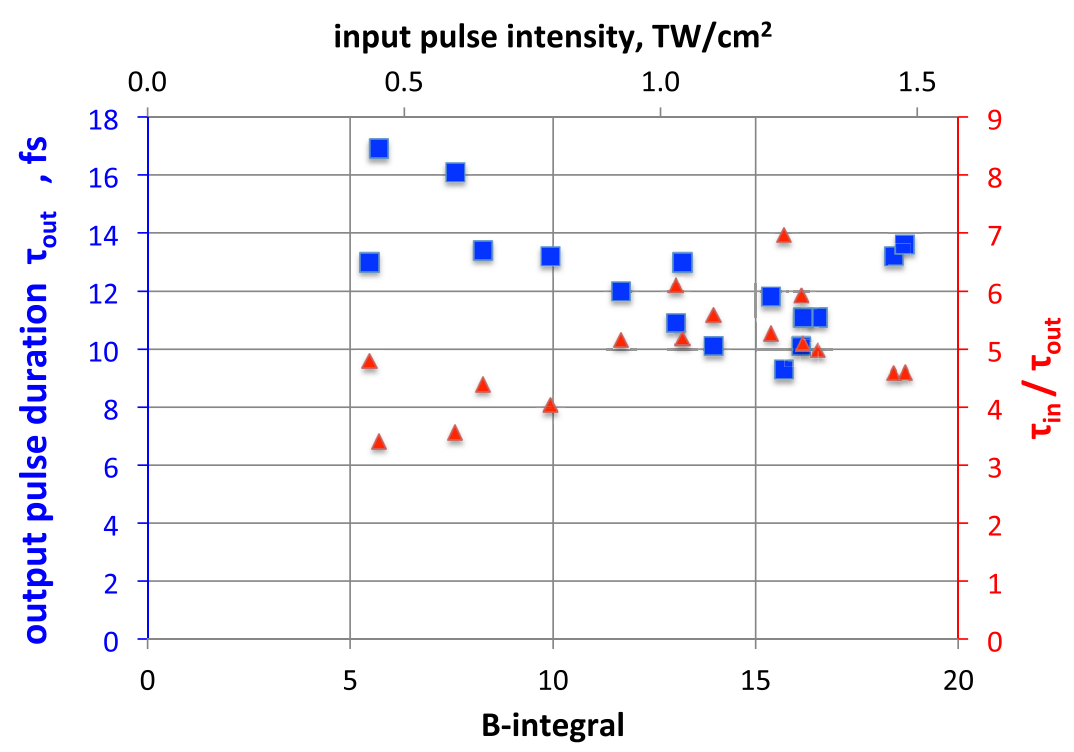

Figure 5. Output pulse duration $\tau_{\text {out }}$ (blue) and pulse compression factor $F_{\tau}=\tau_{\text {in }} / \tau_{\text {out }}($ red $)$ at $\alpha=-200 \mathrm{fs}^{2} ; \tau_{\text {in }}=55, \ldots, 67 \mathrm{fs}$.

The experimental data for $\tau_{\text {in }}=55, \ldots, 67$ fs obtained at $\alpha=-200 \mathrm{fs}^{2}$ are presented in Figure 5 . The peak power $P_{\text {out }}$ for a compressed pulse with $\tau_{\text {out }}=10 \mathrm{fs}$ was $1.5 \mathrm{PW}$ (we took into account the $20 \%$ losses associated with a lower intensity at the beam periphery and the respective larger $\tau_{\text {out }}$ ). Despite the spread in the values in Figure 5, we can claim that at $\alpha=-200 \mathrm{fs}^{2}$ there exists an optimal value of the $B$-integral (of the order of 15 ) at which $\tau_{\text {out }}$ is minimal and $F_{\tau}$ is maximal. With a further increase in $B$, the input pulse spectrum is broadened but, despite this fact, $\tau_{\text {out }}$ increases as $\left|\alpha_{\text {opt }}\right|$ is already less than $200 \mathrm{fs}^{2}$, that is, CMs have redundant dispersion, as a result of which the output pulse is negatively chirped. At the same time, Figure 5 demonstrates that in a wide range of $B$-integral values from 11 to 19 , for the same CMs set $\left(\alpha=-200 \mathrm{fs}^{2}\right)$, all the shots are within the interval of $\tau_{\text {out }}$ from 9.3 to 13.6 fs and $F_{\tau}$ from 4.6 to 7 . From the practical point of view, this spread is not very large and can be made even less if the input pulse has more stable duration and spectral phase.

Despite the huge values of the $B$-integral, no damage was observed either in KDP or in CMs, that is, smallscale self-focusing was not a significant issue. One of the reasons for this is a significant shift of the maximum of the increment of self-focusing instability to the region of high spatial frequencies, which results in a reduced noise power in the region of the maximal increment due to a decrease in the spectral density of the noise ${ }^{[31]}$ and beam self-filtering at free propagation ${ }^{[18,19,31,32]}$. Another possible reason is the convective nature of the self-focusing instability at pulse duration commensurate with 10 field periods ${ }^{[33,34]}$. 
The suppression of small-scale self-focusing is discussed in more detail in Ref. [3].

Note that further power scaling may be limited by damage threshold which strongly depends on the polishing and coating quality. Being a water-soluble crystal, KDP requires more efforts for both polishing and coating compared with silica. In addition, there could be crystal degradation effects on a longer term. This could limit the use of KDP in high peak power femtosecond lasers with high repetition rate.

Thus, we have experimentally demonstrated that KDP can give results similar to $\left(\tau_{\text {in }} / \tau_{\text {out }}>6\right)$, and even a little better $\left(\tau_{\text {out }}=10 \mathrm{fs}\right)$ than those we have recently obtained with silica $^{[17]}$, with the superiority of KDP being still more pronounced for small $B$-integrals $(B=5, \ldots, 9)$ (see Figure 4). In addition, KDP has a number of other advantages. First, for KDP the $\alpha_{\text {opt }}$ values are smaller (Figures 1(a) and 4). This allows a smaller number of mirrors to be used or the same number of mirrors but with lower dispersion, which are easier to produce. Second, for KDP the value of $\alpha_{\mathrm{opt}}$ changes less with the variation of the $B$-integral (Figure 4). This makes it possible to use one set of CMs for a wide range of intensities, as demonstrated in Figure 5. Third, KDP anisotropy enables controlling linear and nonlinear properties of the material by choosing crystal orientation that is determined by the angles $\theta$ and $\varphi$. Like in any uniaxial crystal, the linear refractive index (and, hence, $k_{2}$ and $k_{3}$ ) of an ordinary wave depends neither on $\theta$ nor on $\varphi$. However, similarly to any tetragonal crystal of $\overline{4} 22$ symmetry, $n_{2}$ does not depend on $\theta$, but is dependent on $\varphi^{[35]}$, and $n_{2}$ maxima and minima are, respectively, at $\varphi=0+\pi \mathrm{m} / 2$ and $\varphi=\pi / 4+\pi m / 2$ ( $m$ is an integer). Thus, for example, by rotating a $z$-cut crystal $(\theta=0)$ around the $z$-axis it is possible to continuously change the $B$-integral by about a factor of 1.5: $n_{2}(\theta=0, \varphi=0)=4.6 \times 10^{-16} \mathrm{~cm}^{2} / \mathrm{W}$, and $n_{2}(\theta=0$, $\varphi=\pi / 4)=3.3 \times 10^{-16} \mathrm{~cm}^{2} / \mathrm{W}^{[36]}$. Still more opportunities are provided by an extraordinary wave for which $k_{2}$ and $k_{3}$ depend on $\theta$, and $n_{2}$ depends on both $\theta$ and $\varphi$. The $n_{2}(\theta$, $\varphi)$ function can be found in Ref. [35]. The functions $k_{2}(\theta)$ and $k_{3}(\theta)$ can be readily obtained from the known expression for $n_{\mathrm{e}}(\theta)$ and from the Sellmeier equations ${ }^{[30]}$. Thus, for an extraordinary wave, dispersion and nonlinearity can be controlled independently by varying $\theta$ and $\varphi$, respectively. Note that it is also possible to use a KDP crystal isomorph: a DKDP crystal whose dispersion strongly differs from KDP dispersion $^{[37]}$. The optimization of the parameters $k_{2}, k_{3}$, and $n_{2}$ will be considered in a separate paper.

\section{Conclusion}

In our studies of the nonlinear compression of high-power laser pulses (TFC, CafCA, post-compression) after SPM in a KDP crystal we have obtained the pulse compression factor $\tau_{\text {in }} / \tau_{\text {out }}>6$ with the compressed pulse duration $\tau_{\text {out }}=10 \mathrm{fs}$, which corresponds to a peak power of $1.5 \mathrm{PW}$. To the best of the authors' knowledge, $10 \mathrm{fs}$ is the shortest duration of all present-day petawatt lasers worldwide. It is important to note that the experiments were carried out for the $B$ integral values from 5 to 19 , with no damage to the optical elements, which indicates that small-scale self-focusing is insignificant. Analogous results were recently obtained by our team using silica ${ }^{[17]}$. However, as compared with silica, KDP has several advantages: (i) a larger pulse compression factor $\tau_{\text {in }} / \tau_{\text {out }}$, especially for $B=5, \ldots, 9$; (ii) smaller absolute values of CMs dispersion $\alpha_{\text {opt }}$; (iii) smaller changes in $\alpha_{\text {opt }}$ with the variation of the $B$-integral; and (iv) a possibility to control both linear and nonlinear properties of the medium by choosing the orientation of the crystal's optical axis and the radiation polarization.

Taking into account the obtained results and the undoubted merits of nonlinear compression (simplicity, low cost, negligible pulse energy losses, and applicability to any high-power laser), we predict further development of this approach toward multipetawatt power and single-cycle pulse duration simultaneously.

\section{Acknowledgment}

The work was supported by the Center of Excellence "Center of Photonics" funded by the Ministry of Science and Higher Education of the Russian Federation, agreement No. 075-152020-906 and by the European Commission, grant agreement \#871072-CREMLINplus.

\section{References}

1. C. Danson, J. Bromage, T. Butcher, J.-C. Chanteloup, E. Chowdhury, A. Galvanauskas, L. Gizzi, C. Haefner, J. Hein, D. Hillier, N. Hopps, Y. Kato, E. Khazanov, R. Kodama, G. Korn, R. Li, Y. Li, J. Limpert, J. Ma, C. H. Nam, D. Neely, D. Papadopoulos, R. Penman, L. Qian, J. Rocca, A. Shaykin, C. Siders, C. Spindloe, S. Szatmári, R. Trines, J. Zhu, P. Zhu, and J. Zuegel, High Power Laser Sci. Eng. 7, e54 (2019).

2. G. Mourou, S. Mironov, E. Khazanov, and A. Sergeev, Eur. Phys. J. Spec. Top. 223, 1181 (2014).

3. E. A. Khazanov, S. Y. Mironov, and G. Mourou, Phys.-Usp. 62, 1096 (2019).

4. P. Balla, A. B. Wahid, I. Sytcevich, C. Guo, A.-L. Viotti, L. Silletti, A. Cartella, S. Alisauskas, H. Tavakol, U. GrosseWortmann, A. Schönberg, M. Seidel, A. Trabattoni, B. Manschwetus, T. Lang, F. Calegari, A. Couairon, A. L'Huillier, C. L. Arnold, I. Hartl, and C. M. Heyl, Opt. Lett. 45, 2572 (2020).

5. R. A. Fisher, P. L. Kelley, and T. K. Gustajson, Appl. Phys. Lett. 14, 140 (1969).

6. A. Laubereau, Phys. Lett. 29A, 539 (1969).

7. C. V. Shank, R. L. Fork, R. Yen, R. H. Stolen, and W. J. Tomlinson, Appl. Phys. Lett. 40, 761 (1982).

8. M. Nisoli, S. D. Silvestri, and O. Svelto, Appl. Phys. Lett. 68, 2793 (1996).

9. C. Rolland and P. B. Corkum, J. Opt. Soc. Am. B 5, 641 (1988).

10. V. Chvykov, C. Radier, G. Cheriaux, G. Kalinchenko, V. Yanovsky, and G. Mourou, in Conference on Lasers and Electro-Optics (CLEO)/Quantum Electronics and Laser Science Conference (QELS) (IEEE, 2010). 
11. S. Y. Mironov, V. N. Ginzburg, I. V. Yakovlev, A. A. Kochetkov, A. A. Shaykin, E. A. Khazanov, and G. A. Mourou, Quantum Electron. 47, 614 (2017).

12. S. K. Lee, J. Y. Yoo, J. I. Kim, R. Bhushan, Y. G. Kim, J. W. Yoon, H. W. Lee, J. H. Sung, and C. H. Nam, in Conference on Lasers and Electro-Optics (CLEO) (IEEE, 2018).

13. V. N. Ginzburg, I. V. Yakovlev, A. S. Zuev, A. A. Korobeinikova, A. A. Kochetkov, A. A. Kuz'min, S. Y. Mironov, A. A. Shaykin, I. A. Shaykin, and A. E. Khazanov, Quantum Electron. 49, 299 (2019).

14. D. M. Farinella, J. Wheeler, A. E. Hussein, J. Nees, M. Stanfield, N. Beier, Y. Ma, G. Cojocaru, R. Ungureanu, M. Pittman, J. Demailly, E. Baynard, R. Fabbri, M. Masruri, R. Secareanu, A. Naziru, R. Dabu, A. Maksimchuk, K. Krushelnick, D. Ros, G. Mourou, T. Tajima, and F. Dollar, J. Opt. Soc. Am. B 36, A28 (2019).

15. V. Ginzburg, I. Yakovlev, A. Zuev, A. Korobeynikova, A. Kochetkov, A. Kuzmin, S. Mironov, A. Shaykin, I. Shaikin, E. Khazanov, and G. Mourou, Phys. Rev. A 101, 013829 (2020).

16. S. Y. Mironov, S. Fourmaux, P. Lassonde, V. N. Ginzburg, S. Payeur, J.-C. Kieffer, E. A. Khazanov, and G. Mourou, Appl. Phys. Lett. 116, 241101 (2020).

17. V. Ginzburg, I. Yakovlev, A. Kochetkov, A. Kuzmin, S. Mironov, I. Shaikin, A. Shaykin, and E. Khazanov, Opt. Express 29, 28297 (2021).

18. S. Y. Mironov, V. V. Lozhkarev, V. N. Ginzburg, I. V. Yakovlev, G. Luchinin, A. A. Shaykin, E. A. Khazanov, A. A. Babin, E. Novikov, S. Fadeev, A. M. Sergeev, and G. A. Mourou, IEEE J. Sel. Top. Quantum Electron. 18, 7 (2010).

19. S. Mironov, V. Lozhkarev, G. Luchinin, A. Shaykin, and E. Khazanov, Appl. Phys. B 113, 147 (2013).

20. V. N. Ginzburg, I. V. Yakovlev, A. S. Zuev, A. P. Korobeynikova, A. A. Kochetkov, A. A. Kuzmin, S. Y. Mironov, A. A. Shaykin, I. A. Shaikin, and E. A. Khazanov, Quantum Electron. 50, 331 (2020).
21. M. Stanfield, N. F. Beier, S. Hakimi, H. Allison, D. Farinella, A. E. Hussein, T. Tajima, and F. Dollar, Opt. Express 29, 9123 (2021).

22. A. A. Voronin, A. M. Zheltikov, T. Ditmire, B. Rus, and G. Korn, Opt. Commun. 291, 299 (2013).

23. V. N. Ginzburg, A. A. Kochetkov, I. V. Yakovlev, S. Y. Mironov, A. A. Shaykin, and E. A. Khazanov, Quantum Electron. 46, 106 (2016).

24. Z. Li, Y. Kato, and J. Kawanaka, Sci. Rep. 11, 151 (2021).

25. S. Y. Mironov, M. V. Starodubtsev, and E. A. Khazanov, Opt. Lett. 46, 1620 (2021).

26. E. A. Khazanov, Quantum Electron. 51, 433 (2021).

27. E. Khazanov, Opt. Express 29, 17277 (2021).

28. J. Buldt, M. Muller, R. Klas, T. Eidam, J. Limpert, and A. Tunnermann, Opt. Lett. 42, 3761 (2017).

29. V. V. Lozhkarev, G. I. Freidman, V. N. Ginzburg, E. V. Katin, E. A. Khazanov, A. V. Kirsanov, G. A. Luchinin, A. N. Mal'shakov, M. A. Martyanov, O. V. Palashov, A. K. Poteomkin, A. M. Sergeev, A. A. Shaykin, and I. V. Yakovlev, Laser Phys. Lett. 4, 421 (2007).

30. F. Zernike, J. Opt. Soc. Am. 54, 1215 (1964)

31. V. N. Ginzburg, A. A. Kochetkov, S. Y. Mironov, A. K. Potemkin, D. E. Silin, and E. A. Khazanov, Radiophys. Quantum Electron. 62, 849 (2020).

32. V. N. Ginzburg, A. A. Kochetkov, A. K. Potemkin, and E. A. Khazanov, Quantum Electron. 48, 325 (2018).

33. A. A. Balakin, A. G. Litvak, V. A. Mironov, and S. A. Skobelev, J. Opt. 19, 095503 (2017).

34. A. A. Balakin, A. V. Kim, A. G. Litvak, V. A. Mironov, and S. A. Skobelev, Phys. Rev. 94, 043812 (2016).

35. P. S. Banks, M. D. Feit, and M. D. Perry, J. Opt. Soc. Am. B 19, 102 (2002).

36. I. A. Kulagin, R. A. Ganeev, R. I. Tugushev, A. I. Ryasnyansky, and T. Usmanov, J. Opt. Soc. Am. B 23, 75 (2006).

37. V. V. Lozhkarev, G. I. Freidman, V. N. Ginzburg, E. A. Khazanov, O. V. Palashov, A. M. Sergeev, and I. V. Yakovlev, Laser Phys. 15, 1319 (2005). 